\title{
Influence of Spatial Correlations on the Lasing Threshold of Random Lasers
}

\author{
Michael Patra \\ Laboratory for Computational Engineering, Helsinki University of Technology, P. O. Box 9203, 02015 HUT, Finland
}

\begin{abstract}
The lasing threshold of a random laser is computed numerically from a generic model. It is shown that spatial correlations of the disorder in the medium (i. e., dielectric constant) lead to an increase of the decay rates of the eigenmodes and of the lasing threshold. This is in conflict with predictions that such correlations should lower the threshold. While all results are derived for photonic systems, the computed decay rate distributions also apply to electronic systems.
\end{abstract}

In the theory of disordered media two important regimes, diffusive and localised, are distinguished [1]. In the diffusive regime (for weak or moderate disorder), eigenstates are extended and efficient transport is possible. In the localised regime (for strong disorder), eigenstates become localised and transport is strongly inhibited. Many experimental findings for random lasers are more consistent with the assumption of a lasing mode that is localised while a direct experimental analysis of the sample shows that it is in the diffusive regime.

To determine whether a sample is in the localised or in the diffusive regime, a transport property is measured. The most efficient way to achieve this is to check for the rounding of the backscattering cone [2]. Such a rounding is not reported from experiments 3, 4]. Transport is, however, dominated by extend eigenstates, and the simultaneous existence of a few localised eigenstates in a sample in the diffusive regime, i. e., that is on average diffusive, would not be noticed 5]. Such localised modes have recently been detected experimentally in a diffusive sample 6].

The important question is to explain under which conditions such localised eigenstates can exist in a diffusive sample. [These states have been termed anomalously localised states (ALS) or prelocalised states. For a recent review see Ref. 7.] One-dimensional disordered systems are always in the localised regime, i. e., they can never show diffusive behaviour. Theoretical studies on such systems thus cannot give information on the interplay between extended and localised modes. The situation is different in two- and three-dimensional samples. Twodimensional samples shorter than the localisation length behave similarly to three-dimensional samples, and one is allowed to replace three-dimensional systems by their computationally cheaper two-dimensional counterparts.

The computational cost of treating a two-dimensional sample is significantly higher than for a one-dimensional sample, and only few studies have been published [8]. Reference 5 models the scatterers in the disordered media as dipoles, Ref. 9 studies circular particles using the finite-difference time-domain (FDTD) method. Both publications do not state explicitly whether their samples are in the diffusive or in the localised regime but the parameters given strongly suggest that the samples are in the localised regime.

The only publication so far on the interplay between diffusive and localised eigenstates inside a diffusive sample seems to be Ref. 10. There it was estimated that lo- calised states become exponentially more frequent when the disorder inside the sample is spatially correlated. Motivated by the picture of photons travelling in closed loop inside a ring-shape structure [3], they study a ring-shaped area of higher dielectric constant. This is a very special situation, and it is not obvious how characteristic such a special situation is for the entire behaviour. (It should be noted that the opposite effect, namely that in a localised sample a few modes become extended when spatial correlations in the disorder are introduced, is well-understood, see e.g. Ref. 11.)

In this paper we will study this problem from a more generic approach. The lasing threshold of a sample is determined by the decay rates of the eigenstates of the system since the loss (=decay) of photons in the mode has to be compensated by pumping if the sample is to start lasing action. Following the approach of Ref. 12 we numerically compute the decay rate distribution of a two-dimensional sample on a suitable grid. (Earlier work on the lasing threshold of chaotic cavities [13] cannot be applied since by construction all eigenstates are extended 1].) We improve on previous work by including spatial correlations.

We use the Anderson Hamiltonian which describes the motion of an uncharged particle in a spatially varying potential. The results can directly be applied also to photonic systems since the Helmholtz equation with a spatially varying dielectric constant has the same form. The sample is discretised with lattice spacing $\Delta$, where for electronic systems $\Delta=\pi / k_{\mathrm{F}}\left(k_{\mathrm{F}}\right.$ is the wave vector at the Fermi level) and for photonic systems $\Delta=2 \lambda / \pi$ ( $\lambda$ is the wave length of the light). This is a natural choice in that there is then one propagating mode per transversal lattice point, and the width of the sample is best measured in terms of the number $N$ of propagating modes.

Transport is modelled by nearest-neighbour hopping with rate 1 . (The results are easily adapted to arbitrary speed $c$ of transport.) With a spatially varying potential $P(x, y)$ the Hamiltonian for a sample of length $L=\tilde{L} \Delta$ becomes [12]

$$
\begin{aligned}
& \mathcal{H}_{(x, y),\left(x^{\prime}, y^{\prime}\right)}=\delta_{x x^{\prime}} \delta_{y y^{\prime}}\left[P(x, y)-i\left(\delta_{1 y}+\delta_{L y}\right)\right] \\
& +\delta_{y y^{\prime}}\left(\delta_{x+1, x^{\prime}}+\delta_{x-1, x^{\prime}}\right)+\delta_{x, x^{\prime}}\left(\delta_{y+1, y^{\prime}}+\delta_{y-1, y^{\prime}}\right)
\end{aligned}
$$

with $x=1, \ldots, \tilde{L}$ and $y=1, \ldots, N$. The imaginary part of $\mathcal{H}$ models coupling of the sample to the outside 
where we assume that we operate at the centre of the conduction band.

The spatial correlations are assumed to fall of exponentially such that $P(x, y)$ takes on random values, normaldistributed with zero mean and correlator

$$
\left\langle P(\vec{r}) P\left(\vec{r}^{\prime}\right)\right\rangle=w^{2} \exp \left(-\frac{\left|\vec{r}-\vec{r}^{\prime}\right|}{R_{\mathrm{c}}}\right) .
$$

$w$ measures the strength of the disorder, and $R_{\mathrm{c}}$ is the correlation radius. Since we need to generate a large number of mutually correlated random numbers, a Fourier based method has to be employed 14].

The eigenvalues of the matrix $\mathcal{H}$ correspond to the (quasi-)eigenmodes of the system. Their real part $\omega$ gives the energy (or, for photonic systems, the frequency) of the mode, and their imaginary part $\gamma$ the decay rate [15]. We thus have an eigenvalue problem of a non-Hermitian complex symmetric matrix but an eigensolver specifically adopted to this structure exists [12]. Even with this efficient eigensolver, this is still a numerically expensive task, and it is impossible to analyse so many samples that there would be no more noise in the results.

While the model is described in terms of the disorder strength $w$, contact with experiments or analytical theories is best made by introduction of the mean-free path $l$. It can be computed from the length-dependence of the transmission probability $T$ through the sample. In the diffusive regime, $l \lesssim L \ll N l$, it is given by [1]

$$
\frac{1}{T}=1+\frac{L}{l} .
$$

The transmission probability has been computed using the method of recursive Green's functions 16] for variable disorder strength $w$ and correlation length $R_{\mathrm{c}}$. We determined the mean-free path by fitting the numerically computed $T(L)$ to this functional form self-consistently in the interval $[l ; 10 l]$. (Picking some other interval, e.g. $[0 ; l]$, changed the result only by about $1 \%$.) The rescaled results are depicted in Fig. 1 for both $N=51$ and $N=81$, i. e., for samples of different width. As the figure shows, both sets of curves are almost identical, thereby demonstrating that we are operating in the wide-sample regime. The mean-free path increases significantly as $R_{\mathrm{c}}$ increases. This is immediately obvious since with increasing $R_{\mathrm{c}}$ the potential changes less within a given distance; hence, there is less scattering.

We would like to point out two "curiosities". The numerical data suggest that the mean-free path $l$ factorises as $l\left(w, R_{\mathrm{c}}\right)=f_{1}(w) f_{2}\left(R_{\mathrm{c}}\right)$. We did not manage to find an explanation for this observation. Furthermore, the mean-free path seems to scale as $l \propto w^{-1.71}$ where 1.71 is a numerical parameter. For uncorrelated random order that is uniformly distributed in the interval $[-w ; w]$, a scaling $l \propto w^{-1.5}$ was found numerically [12]. An analytical theory is available only for one-dimensional systems in the limit $w \rightarrow 0$ where $l \propto 1 / w^{2}$ is found [17], so that a universal scaling for finite $w$ might not exist at all.

The increase of $l$ with increasing $R_{\mathrm{c}}$ poses a problem for a systematic study of the effects of correlations: One

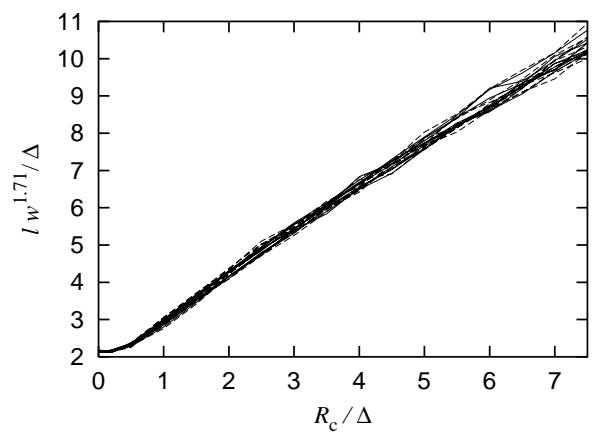

FIG. 1: Numerically computed rescaled mean-free path $l$, depending on the disorder strength $w$ and the correlation radius $R_{\text {c }}$ (in units of the lattice spacing $\Delta$ ). The solid lines are for samples of width $N=51$, the dashed lines for samples of width $N=81$. Samples were computed with $w$ in steps of 0.1 and $R_{\mathrm{c}}$ in steps of $0.5 \Delta$ (plus the value $R_{\mathrm{c}}=0.2 \Delta$ ). By rescaling $l \rightarrow l w^{1.71}$ we can demonstrate the apparent scaling $l \propto w^{-1.71}$ and the factorisation $l\left(w, R_{\mathrm{c}}\right)=f_{1}(w) f_{2}\left(R_{\mathrm{c}}\right)$.

has to decide whether to compare samples with identical $l$ (and thus variable $w$ ) or samples with identical $w$ (and thus variable $l$ ). The final results must depend (apart from trivial prefactors) only on the ratios $L / l$ and $R_{\mathrm{c}} / l-$ not on any of those quantities separately. This decision is thus "only" one of numerical efficiency and minimisation of finite-size effects.

For most of our simulations, we have decided to keep $l$ constant at $l=12.5 \Delta$. For each value of $R_{\mathrm{c}}$, the needed value for $w$ was determined by interpolation of the numerical data presented in Fig. 10 The choice of constant $l$ offers the advantage that, even if $R_{\mathrm{c}}$ is changed, samples with identical "physical" length $L / l$ occupy the same number of lattice points, and thus need the same amount of numerical work. (For constant "physical" length $L / l$, the needed computing time scales as $\mathcal{O}\left(l^{2}\right)$. With constant $w$, this would impose severe restrictions on the range of $R_{\mathrm{c}}$ that could be treated.)

We have computed the decay rates for samples of width $N=50$ for length $L / l=1,2,3,4,5,6,9,12,15,18$, and correlation radius $R_{\mathrm{c}} / \Delta=0.0,0.2,0.5,1.0,1.5, \ldots, 7.5$. For each set of parameters, approximately 2000 samples were generated. The maximum value of $L$ is limited because we are interested in the diffusive regime, hence $L$ has to be sufficiently smaller than the localisation length $L_{\text {loc }}=(N+1) l / 2$. We did not consider larger values of $R_{\mathrm{c}}$ than $7.5 \Delta$ since the sample should be much wider than the characteristic length scale of the disorder. Otherwise the sample would effectively become one-dimensional.

To check the results, we have computed the decay rate distribution also for $N=80$ for a few selected values of $L / l$ and $R_{\mathrm{c}}$. To complement the other simulations, we have kept $w$ constant. As explained above, this implies that we could only include $R_{\mathrm{c}} \leq 2 \Delta$.

Following the approach introduced in Ref. 12 for samples in the diffusive regime, we fit the numerically com- 


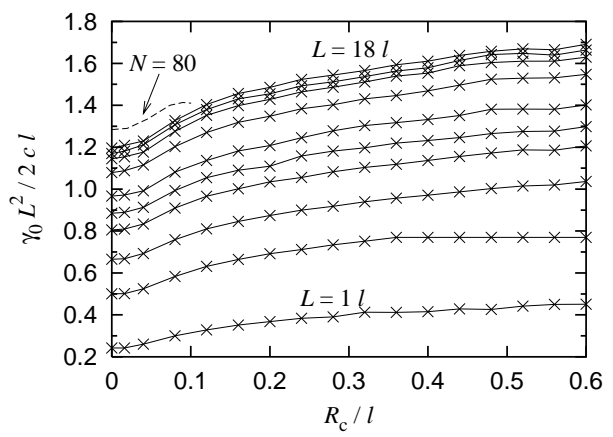

FIG. 2: Characteristic decay rate $\gamma_{0}$ as a function of sample length $L$ and correlation radius $R_{\text {c }}$ (for samples of width $N=$ 50). The dashed line is for the control simulations with $N=$ 80 and $L=25 l$.

puted decay rate distribution to the functional form

$$
\mathcal{P}(\gamma)=\frac{\gamma_{0}^{2}}{\gamma^{2}}\left[1-Q\left(M+1, \frac{M \gamma}{\gamma_{0}}\right)\right]
$$

where the fitting parameters $M$ and $\gamma_{0}$ depend on $N$, $L$ and $R_{\mathrm{c}}$, and $Q(a, x) \equiv \Gamma(a, x) / \Gamma(a)$ is the regularised Gamma function .

All numerically computed histograms fit well to the form (4). The dependence of $P(\gamma)$ onto $M$ is only weak for $M \gg 1$, making a precise determination of $M$ difficult. Within this error limit, we did not find a significant dependence of $M$ on $R_{\mathrm{c}}$, and $\mathrm{M}$ is approximately given by the $R_{\mathrm{c}}=0$ result $M=N /[1+L /(6 l)][12]$.

The fitting parameter $\gamma_{0}$, marking the typical value of the decay rates, can be determined to much better precision. $\gamma_{0}$ is much more important for the lasing threshold than $M$, so the limited precision of $M$ does not pose a problem. The determined $\gamma_{0}$ is shown in Fig. 2]

For $R_{\mathrm{c}}=0, \gamma_{0} L^{2}$ seems to approach a constant value as $L$ is increased. This value is about $20 \%$ larger than the value $1 /(2 c l)$ found numerically for equi-distributed disorder in the interval $[-w ; w]$ 12]. Trying to approach the limit $L \rightarrow \infty$ numerically is not possible since then the sample would become localised.

The important conclusion from Fig. 2 is that for samples of arbitrary length, the introduction of correlations in the disorder leads to an increase of the decay rates. This increase is quick as $R_{\mathrm{c}}$ is increased starting from 0 , and becomes slower for large $R_{\mathrm{c}}$. The same behaviour is seen in the control simulations with $N=80$ and fixed $w$ (and thus variable $l$ ).

Until now, all results are valid for both electronic and photonic systems. Now we will specialise to random lasers. The light inside a random laser is amplified by a laser dye. This dye is able to amplify light within a certain range of frequencies, so only $K \gg 1$ eigenmodes out of all eigenmodes of the system are amplified. This number varies only slightly between different realisations of the same ensemble due to an effect known as spectral rigidity [1]. The lasing threshold is given by the smallest decay rate out of the $K$ modes within the amplification window [18]. This is immediately obvious since the lasing threshold is passed when photons are created faster than they can decay (=escape from the sample).

There are two different approaches to computing the lasing threshold of a random laser. The direct approach is to compute the eigenmodes of a certain number of realisations of the disordered systems, then for each realisation to determine the smallest decay rate inside the amplification window, and finally collect statistics for those values. Since this process yields only a single datum per sample, a very large number of realisations needs to be computed to arrive at data of sufficient quality. The average lasing threshold determined in this way is depicted in Fig. 3 as dashed line.

Frequently more efficient is the second approach where one starts with the computation of the distribution $P(\gamma)$ of the individual decay rates. The intermediary result is either a numerical histogram, or, by fitting the histogram to an analytical form, a distribution function that can be evaluated directly for arbitrary argument. We adopt the latter and use Eq. (4) ) together with the values of $M$ and $\gamma_{0}$ computed by fitting.

The distribution $P_{1}\left(\gamma_{1}\right)$ of the lasing threshold is the distribution of the smallest value out of $K$ values, each distributed according to $P(\gamma)$. This assumes that the decay rates of different modes are uncorrelated. For $K \gg$ 1 this seems logical but to our best knowledge no explicit check of this assumption has been published so far. As a side-effect of our computations, we will fill this gap.

$P_{1}\left(\gamma_{1}\right)$ is difficult to evaluate numerically for $K \gg 1$ since it is sharply peaked. The position $\gamma_{\mathrm{m}}$ of the maximum of $P_{1}$ is immediately seen to be given by

$$
0=\frac{d P\left(\gamma_{\mathrm{m}}\right)}{d \gamma_{\mathrm{m}}}\left[1-\int_{0}^{\gamma_{\mathrm{m}}} P\left(\gamma^{\prime}\right) d \gamma^{\prime}\right]-(K-1)\left[P\left(\gamma_{\mathrm{m}}\right)\right]^{2}
$$

Since $P_{1}$ is that sharply peaked, $\gamma_{\mathrm{m}}$ already contains all the relevant information, and nothing relevant is lost by not computing the entire distribution. The lasing threshold computed from Eq. (5), after inserting the fit-

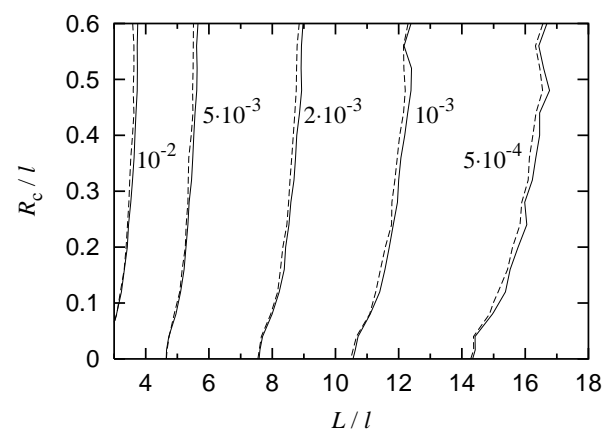

FIG. 3: Comparison of the average of the lasing threshold, directly computed from the numerical data (solid line), and the most likely lasing threshold computed from the distribution of the individual decay rates (dashed line). Both lines have been computed from the same samples, explaining the correlation of the noise in the two sets of lines. 
ting parameters $M$ and $\gamma_{0}$ computed from the numerical histograms into Eq. (4), is shown as solid line in Fig. [3]

From the figure, two important conclusions can be drawn. First, the lasing threshold computed via the two separate methods agrees well. (The "noise" of the two sets of curves is correlated since the same raw data was used as input for both methods.) This means that the decay rates of different modes indeed are uncorrelated. Furthermore, also fitting the numerical histogram to the form (44) is a valid procedure.

The second conclusion - the heart of this paper - is that introducing spatial correlations into the disorder of a random laser increases the lasing threshold, in contradiction to predictions [10].

In this paper, we have thus arrived at two related but not identical - results. We have shown that the decay rates increase if spatial correlations of the disorder are introduced. The computed decay rate distribution possesses the same form, just with different parameter, as earlier observed for diffusive samples with uncorrelated disorder 12. This first result means that the "typical" eigenstates become more lossy.

Our second result is that also the lasing threshold increases. This means that also the "special" eigenstates with lower-than-average loss, which are selected by mode competition to become the lasing modes, become more lossy. Even though we did not directly compute the spatial extend of the eigenstates, this still clearly demonstrates that no (pre-)localised eigenstates are formed by the introduction of spatial disorder. We thus fail to observe the prediction that such states should be created [10].
There are several explanations for the difference between our results and Ref. 10. One explanation is that a single ring-shaped area of increased dielectric constant does lead to the formation of a localised state, as suggested by the authors, but the influence of the disorder around that ring-shaped area significantly reduces this effect. Another, equally likely, explanation is that in our simulations we are only able to treat samples of finite size, with a finite number of eigenstates. The creation of a localised state may be an event that is so rare the we fail to see such an event occur in our finite-size simulations. On the other hand, the typical length scale is given by the area per lasing mode, measured experimentally to be a few $10 \mu \mathrm{m}^{2}$ in two-dimensional $\mathrm{ZnO}$ films [19], and our samples are larger than this.

To give a definite answer on whether spatial correlations can explain the formation of localised states, more numerical studies are needed, preferably using different methods. Specialised but numerically efficient models $[\underline{5}$ cannot incorporate spatial correlations of the dielectric constant. Two-dimensional FDTD simulations have already been used to describe random lasers [9]. They need to make only minimal assumptions and they can be extended to include arbitrary spatial correlations. FDTD simulations thus might be a good candidate but diffusive samples need to be larger than the localised samples studied so far. Given that FDTD is computationally very expensive, it is not obvious to us whether this would still be numerically feasible.

This work was supported by the European Union Marie Curie fellowship HPMF-CT-2002-01794.
[1] C. W. J. Beenakker, Rev. Mod. Phys. 69, 731 (1997).

[2] F. J. P. Schuurmans, M. Megens, D. Vanmaekelbergh, and A. Lagendijk, Phys. Rev. Lett. 83, 2183 (1999).

[3] H. Cao et al., Phys. Rev. Lett. 82, 2278 (1999); Phys. Rev. B 59, 15107 (1999); Phys. Rev. E 61, 1985 (2000).

[4] G. van Soest, F. J. Poelwijk, R. Sprik, and A. Lagendijk, Phys. Rev. Lett. 86, 1522 (2001).

[5] A. L. Burin, M. A. Ratner, H. Cao, and R. P. H. Chang, Phys. Rev. Lett. 87, 215503 (2001).

[6] H. Cao, Y. Ling, J. Y. Xu, and A. L. Burin, Phys. Rev. E 66, 025601 (2002).

[7] A. D. Mirlin, Phys. Rep. 326, 259 (2000).

[8] There has been a large number of studies using the diffusion approximation. Using this approximation is numerically very efficient but forbids the existence of localised states. These studies thus cannot explain the interplay between extended and localised eigenstates.

[9] P. Sebbah and C. Vanneste, Phys. Rev. B 66, 144202 (2002).

[10] V. M. Apalkov, M. E. Raikh, and B. Shapiro, Phys. Rev. Lett. 89, 016802 (2002).

[11] F. M. Izrailev and A. A. Krokhin, Phys. Rev. Lett. 82, 4062 (1999).
[12] M. Patra, Phys. Rev. E 67, 016603 (2003).

[13] M. Patra, H. Schomerus, and C. W. J. Beenakker, Phys. Rev. A 61, 23810 (2000); K. M. Frahm et al., Europhys. Lett. 49, 48 (2000); H. Schomerus et al., Physica A 278, 469 (2000).

[14] B. Kozintsev, Ph.D. thesis, University of Maryland (1999).

[15] Actually, the imaginary part of the eigenvalue differs by a factor 2 from the definition of the decay rate. The same factor 2 appears in the definition of the lasing threshold such that those two factors cancel. For ease of writing, we will in the following refer to $\gamma$ as decay rate, not each time mentioning the factor 2.

[16] H. U. Baranger, D. P. DiVincenzo, R. A. Jalabert, and A. D. Stone, Phys. Rev. B 44, 10637 (1991).

[17] B. Kramer and A. MacKinnon, Rep. Prog. Phys. 56, 1469 (1993).

[18] T. S. Misirpashaev and C. W. J. Beenakker, Phys. Rev. A 57, 2041 (1998).

[19] Y. Ling, H. Cao, A. L. Burin, M. A. Ratner, X. Liu, and R. P. H. Chang, Phys. Rev. A 64, 063808 (2001). 